\title{
Dynamics of Nanoparticles in a Supercooled Liquid
}

\author{
Chiara Caronna, ${ }^{1,2}$ Yuriy Chushkin, ${ }^{1}$ Anders Madsen, ${ }^{1, *}$ and Antonio Cupane ${ }^{2}$ \\ ${ }^{1}$ European Synchrotron Radiation Facility, B.P. 220, F-38043 Grenoble, France \\ ${ }^{2}$ Department of Physical and Astronomical Sciences, via Archirafi 36, I-90123, Palermo, Italy \\ (Received 30 July 2007; revised manuscript received 19 December 2007; published 8 February 2008)
}

\begin{abstract}
The dynamic properties of nanoparticles suspended in a supercooled glass forming liquid are studied by $\mathrm{x}$-ray photon correlation spectroscopy. While at high temperatures the particles undergo Brownian motion the measurements closer to the glass transition indicate hyperdiffusive behavior. In this state the dynamics is independent of the local structural arrangement of nanoparticles, suggesting a cooperative behavior governed by the near-vitreous solvent.
\end{abstract}

The glass transition of supercooled liquids [1] is an intriguing phenomenon which continues to receive a considerable amount of interest, both from an experimental and a theoretical point of view. For molecular liquid glass formers, supercooling leads to an increase in rigidity and finally a vitrification due to complex molecular processes which still are poorly understood. It is believed that thermally activated motion (hopping) [2] between different minima configurations of the energy landscape [3] gets important in the supercooled state. The transition to landscape dominated dynamics and dynamical heterogeneity was found to happen at temperatures higher than the calorimetric glass transition temperature $T_{g}[4,5]$. Several studies have been reported where probe molecules were used to investigate the local molecular dynamics of liquid glass formers (see, e.g., Refs. [6,7]), but here we focus on the hydrodynamic behavior of larger tracer particles in a supercooled solvent. X-ray photon correlation spectroscopy (XPCS) was employed to probe the dynamics of silica nanoparticles suspended in the glass-forming liquid propanediol, and the data indicate a transition from Brownian motion to hyperdiffusive behavior as $T_{g}$ is approached.

Sterically stabilized colloidal silica, from EKA Chemicals (16 nm radius) and Duke Scientific (250 nm radius), was delivered suspended in water and has previously been characterized by small angle x-ray scattering (SAXS) $[8,9]$. The water solvent was substituted by 1,2 propanediol (Sigma-Aldrich) using a thorough distillation and centrifugation procedure and the samples were filled into cylindrical quartz capillaries (diam. $\sim 1.5 \mathrm{~mm}$ ) which were sealed to prevent evaporation. The melting temperature of pure propanediol is $T_{m} \sim 245 \mathrm{~K}$ and $T_{g}$ was measured by differential scanning calorimetry [10] to $170 \pm 2 \mathrm{~K}$ for all investigated samples (including pure propanediol). For the $\mathrm{x}$-ray measurements the capillaries were placed in a thermostated $\mathrm{Cu}$ sample holder and good thermal contact was ensured by application of low temperature conducting grease. The sample holder was situated in a vacuum chamber $\left(\sim 10^{-7}\right.$ mbar $)$ operating in the range $\sim 100-400 \mathrm{~K}$ with a stability better than $0.01 \mathrm{~K}$. Static SAXS measurements showed that the size and poly- dispersity of the silica particles and their interactions did not change after the solvent substitution.

The experiments were performed at the Troika beam line ID10A of the European Synchrotron Radiation Facility (ESRF). A collimated $8 \mathrm{keV}$ undulator beam was reflected from a single bounce $\mathrm{Si}(111)$ monochromator and a downstream Si mirror was employed to suppress higher order light. An adjustable aperture was used to select the "coherent part" of the beam. The fringes produced by the aperture scattering were removed by careful positioning of guard slits a few $\mathrm{cm}$ upstream of the sample. A pixelated 2D detector (Medipix-2 [11]) was recording the scattering patterns $\sim 2.3 \mathrm{~m}$ downstream of the sample. The detector consists of $256 \times 256$ pixels each having an area of $55 \times$ $55 \mu \mathrm{m}^{2}$. Each pixel is single photon sensitive and the detector has essentially no dark current because the upper and lower detection limits and gain can be set for each pixel. For every measurement thousands of frames were recorded and subsequently analyzed by the multispeckle XPCS method [12] to obtain a set of temporal correlation functions in the accessible $q$ range.

Figure 1 shows a series of correlation functions taken on a dilute $(\phi \sim 1 \%)$ suspension of $R=250 \mathrm{~nm}$ particles (corresponding to an average interparticle distance of

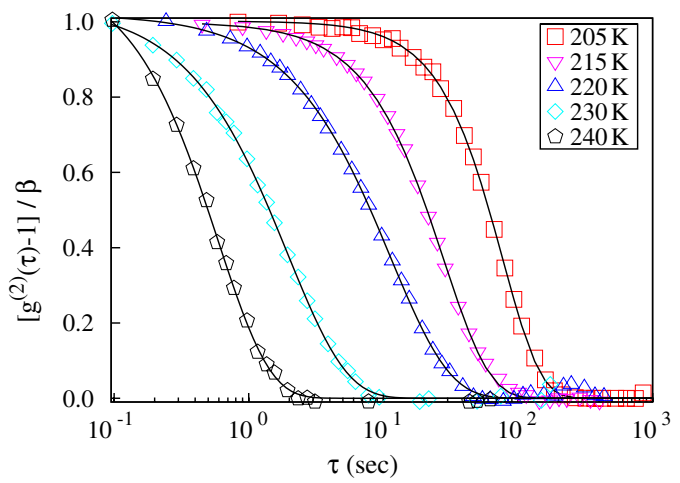

FIG. 1 (color online). Normalized correlation functions (symbols) measured at $q=0.027 \mathrm{~nm}^{-1}$ on a dilute suspension of $250 \mathrm{~nm}$ spheres at different temperatures. Solid lines are fits with the KWW expression. 
about $1800 \mathrm{~nm}$ ) and obviously the dynamics is getting slower as the temperature is lowered. Interestingly, the shape of the correlation functions is also changing (Fig. 1) and this can be quantified by fitting with the Kohlrausch-Williams-Watts (KWW) expression [13]

$$
g^{(2)}(\tau)=\beta \exp \left[-2(\Gamma \tau)^{\gamma}\right]+1
$$

where $\gamma$ is the Kohlrausch exponent, $\Gamma$ is the relaxation rate, and $\beta$ is the contrast. The contrast of the correlation functions obtained in the setup was about $20 \%$ with a slight $q$ dependence as expected for the given sample-detector distance and the sizes of pixels and pinhole. In the investigated temperature range $240-205 \mathrm{~K}$ the KWW expression fits the data well and reveals a transition from simple exponential relaxations $(\gamma=1)$ to compressed exponential behavior $(\gamma>1)$ at low temperatures (Fig. 1).

Parameters extracted from the KWW fits are shown in Fig. 2. At the highest temperatures $(220-240 \mathrm{~K})$ a free diffusive behavior is observed with $\Gamma=D_{0} q^{2}$ and $\gamma=1$; this means that the particles behave like noninteracting Brownian walkers in a Newtonian liquid. The kinetic energy for the motion is delivered by thermal excitations and the friction is due to the particle's size and shape, and the viscosity $\eta$ of the solvent. Assuming spherical particles and a radius of $250 \mathrm{~nm}$ the viscosity of the solvent can be estimated from the fitted values of the free diffusion coefficient $D_{0}$ and one finds $\eta=1.6,5.1$, and $16 \mathrm{~Pa} \cdot \mathrm{s}$ for $T=$ 240,230 , and $220 \mathrm{~K}$, respectively.

Significant deviations from the above picture appear at lower temperatures (215-205 K), as shown in Fig. 2. The change in slope of the dispersion relation from $n \sim 2$ to $n \sim 1$ [Fig. 2(a)] indicates a transition to a ballistic type of motion $(\Gamma \propto q)$. At $205 \mathrm{~K}$ the slope is close to unity and a
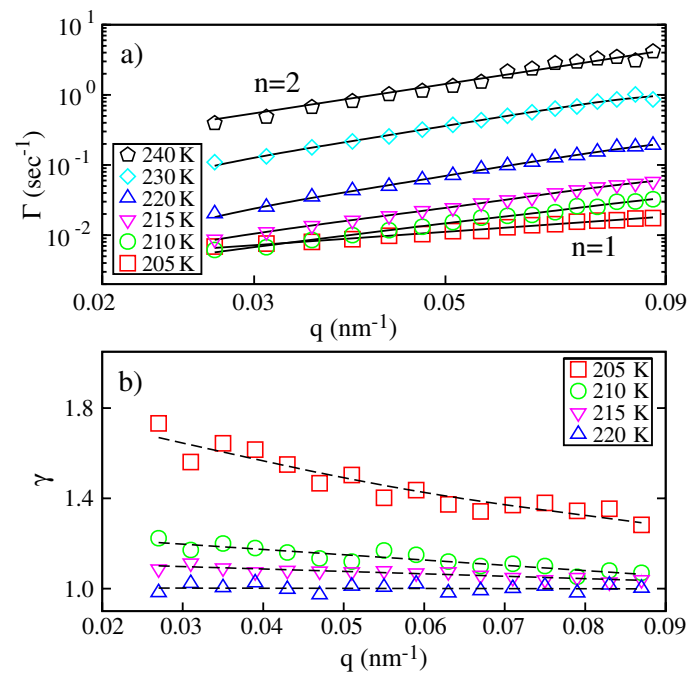

FIG. 2 (color online). (a) $\Gamma(q)$ and (b) $\gamma(q)$ obtained from fits of Eq. (1) to the data. Solid lines are fits with a simple power law $\Gamma \propto q^{n}$; dashed lines are guides for the eye. $\gamma(q)$ is not shown for 230 and $240 \mathrm{~K}$ as it is unity and would overlap the $220 \mathrm{~K}$ data. stagnation of $\Gamma$ at small $q$, i.e., large length scales, is evident. A similar phenomenon has previously been observed in supercooled fragile liquids [14] and associated with dynamical heterogeneity. Moreover, the correlation functions change from simple to compressed exponential decays. For $T<220 \mathrm{~K}$ the exponent $\gamma$ is larger than unity and a clear $q$ dependence is observed [Fig. 2(b)]. In a number of recent experiments, compressed exponential decays with $\gamma>1$ have appeared as a common feature of jammed, nondiffusive systems [15-19] and a microscopic model, based on relaxations of elastic strain deformations as energy source for the particle motion, was proposed [20,21]. Nonequilibrium aging dynamics also seems to be a common property of jamming in soft condensed matter $[15,16,18,19]$ but in the time range covered here (a few hours) we have not observed any aging effects.

Our data indicate that the intermediate scattering functions $f(q, t)=\sqrt{\left(g^{(2)}-1\right) / \beta}$ can be written as

$$
f(q, t) \sim \exp \left[-\left(q^{n} t\right)^{\gamma}\right] .
$$

Such behavior can be described by the continuous time random walk model $[22,23]$ where the displacement of a particle in the time interval $t$ consists of $N$ discrete steps. Hence, the intermediate scattering function is determined by the number of steps $N$, and by the degree of decorrelation $h(q, N)$ they produce, and one can write

$$
f(q, t)=\sum_{N=0}^{\infty} P_{t}(N) h(q, N),
$$

where $P_{t}(N)$ is the probability of $N$ events occurring during the time interval $t$. For simplicity, $P_{t}(N)$ is usually taken as a Poisson distribution $P_{t}(N)=\exp \left(-\Gamma_{0} t\right)\left(\Gamma_{0} t\right)^{N} / N$ ! with $1 / \Gamma_{0}$ as the mean time between events. The decorrelation $h(q, N)$ of the intermediate scattering function can be modeled and it was argued [24,25] that

$$
h(q, N)=\left\langle\exp \left(-i N^{\alpha} \mathbf{q} \cdot \mathbf{R}\right)\right\rangle,
$$

where the averaging is performed over all particles and all orientations of $\mathbf{q}$. The parameter $\alpha$ has a value between 0 and 1 and $\mathbf{R}$ is the particle displacement due to a single step. It is important to note that $\alpha$ determines the nature of the particle motion: if $\alpha=1$ the total displacement due to $N$ steps will be $N \mathbf{R}$, i.e., the particle will move in the same direction (ballistic motion). $\alpha=1 / 2$ defines the special case of Brownian motion while $\alpha<1 / 2$ and $\alpha>1 / 2$ describe the subdiffusion and hyper-diffusion regimes, respectively. To calculate the average in Eq. (4) it only remains to define an appropriate distribution $P(\mathbf{R})$ of particle jumps. Assuming that the dilute suspension of noninteracting particles is analogous to an isotropic gaslike system, a Gaussian distribution of $\mathbf{R}$ is used and one obtains

$$
h(q, N) \simeq \exp \left[-\left(q N^{\alpha} \delta\right)^{2}\right],
$$


where $\delta$ is the average length of a single jump [21]. Equation (5) marks a profound difference with respect to the aforementioned jammed systems: in those cases $P(\mathbf{R})$ was described by the so-called Levy flights $P(\mathbf{R}) \sim$ $R^{-(1+p)}$ with $p=3 / 2$, which implies a maximum value of $3 / 2$ for $\gamma$ in agreement with their experimental results [25]. When $\alpha=1$ the choice of a Gaussian distribution implies $\gamma \rightarrow 2$ for $q \rightarrow 0$ in good agreement with our data [see Fig. 2(b)].

Using Eqs. (3) and (5) the intermediate scattering function $f(t)$ can be calculated as shown in Fig. 3(a) and 3(b) with $\alpha=1$ and the given values of $\delta$ and $\Gamma_{0}$. Fits with the KWW form show an almost perfect agreement, which justifies the use of Eq. (1) to extract $\gamma$ and $\Gamma$ from the correlation functions. Hence, by calculating $f(t)$ from Eq. (3) theoretical curves for $\Gamma(q)$ and $\gamma(q)$ can be generated and used to model the data. For every temperature the fits were performed simultaneously on both data sets $\Gamma(q)$ and $\gamma(q)$ since they are strongly coupled in the model. The measured $q$ dependence of $\Gamma$ and $\gamma$ are shown in Figs. 3(c) and 3(d) together with the best fits, and the obtained parameters imply a change of $\alpha$ from $1 / 2$ (free diffusion) to 1 (ballistic motion) and of $\Gamma_{0}$ from 2.0 to $0.09 \mathrm{~s}^{-1}$ upon cooling from 220 to $205 \mathrm{~K}$. The temperature variations of all fit parameters are shown in Fig. 4. It is noteworthy that while $\delta$ stays roughly unchanged (mean $\sim 8 \mathrm{~nm}$ ) excluding an avalanche-type behavior with increasing particle displacements, $\Gamma_{0}$ and $\alpha$ show an interesting and nontrivial temperature dependence: above $220 \mathrm{~K}$ the dynamics is diffusive with $\alpha=1 / 2$ and an exponentially decreasing $\Gamma_{0}$ caused by the corresponding increase in viscosity as the temperature is lowered. Below $220 \mathrm{~K}$ a fast transition to ballistic motion $(\alpha=1)$ is seen
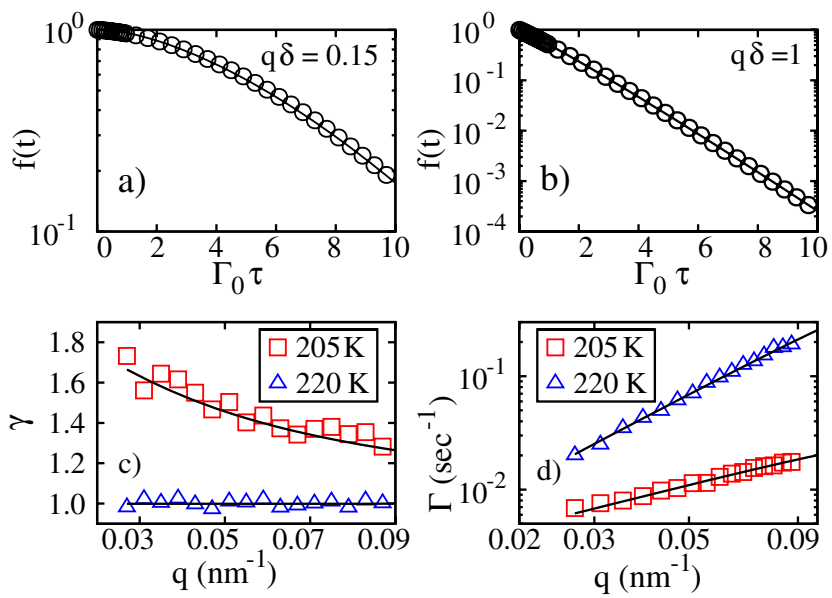

FIG. 3 (color online). (a), (b) Calculated $f(t)$ (symbols) according to Eqs. (3) and (5) at different $q$; solid lines are fits with the KWW form. (c) $\gamma(q)$ and (d) $\Gamma(q)$ data obtained at $205 \mathrm{~K}$ ( $\square$ ) and $220 \mathrm{~K}(\triangle)$; the fits (solid lines) with the model (see text) yield $\left(\delta, \Gamma_{0}, \alpha\right)=(5.4 \mathrm{~nm}, 0.09 \mathrm{~Hz}, 1)$ and $(6.2 \mathrm{~nm}, 2.0 \mathrm{~Hz}, 0.5)$ for 205 and $220 \mathrm{~K}$, respectively. together with a rapid decrease in the jump frequency $\Gamma_{0}$. Around $205 \mathrm{~K}$, however, there are indications that $\Gamma_{0}$ stagnates and hence the translational diffusion of nanoparticles is no longer slowed down according to the increase in solvent viscosity. Attempts to measure below $205 \mathrm{~K}$ failed since signatures of microcracks and possibly crystal formation appeared in the small angle scattering.

The onset of ballistic motion in a system of dilute nanoparticles suggests that the solvent imposes a preferential direction of motion. As a logical consequence one would expect that particles sufficiently close to each other tend to move cooperatively. However, experimental evidence of this idea cannot be obtained with a dilute suspension, in view of the large interparticle distance. For this reason, a concentrated suspension with volume fraction $\phi \sim 20 \%$ was prepared; the solvent was propanediol as before, and to facilitate measurements in the vicinity of the structure factor peak, silica particles of $16 \mathrm{~nm}$ radius were employed (corresponding to an average interparticle distance of about $40 \mathrm{~nm}$ ). A multispeckle XPCS analysis was applied to calculate the correlation functions that were fitted with the KWW form, and the results are shown in Fig. 5. Here, the dispersions $\Gamma$ versus $q$ at the highest temperatures show dips at $q \sim 0.17 \mathrm{~nm}^{-1}$ which correspond to the peak in the structure factor $S(q)$. This so-called "de Gennes narrowing" is a generic feature of diffusing particles in concentrated suspensions demonstrating the slower decay of the most probable density fluctuations due to caging by neighboring particles. Surprisingly, upon cooling this effect vanishes (see Fig. 5) while the peak in $S(q)$ remains at the same position (not shown). This is a clear indication of cooperative behavior induced by the solvent in the same temperature range where the onset of ballistic motion was observed in the dilute sample.

In conclusion, XPCS was used to directly quantify the $q$-resolved hydrodynamics of colloidal nanoparticles suspended in a supercooled molecular glass former. The data unambiguously show the diffusive motion changing into a hyperdiffusive dynamic behavior in the supercooled state. Here, the dynamics is event dominated with a character-

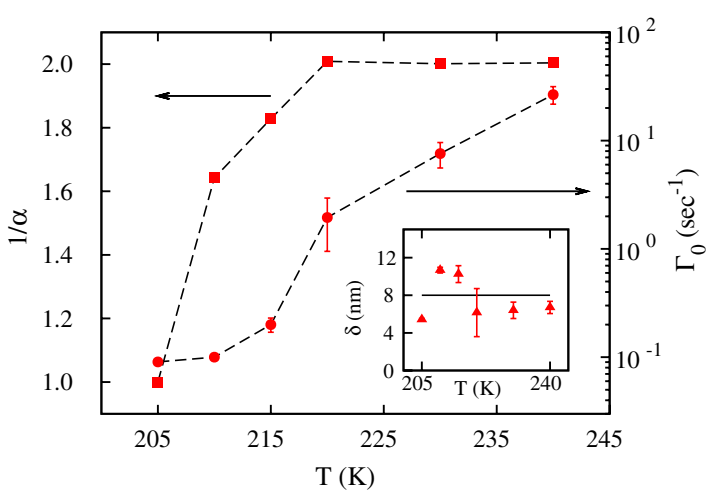

FIG. 4 (color online). Temperature dependence of the fitting parameters $\alpha, \Gamma_{0}$, and $\delta$ (inset). 


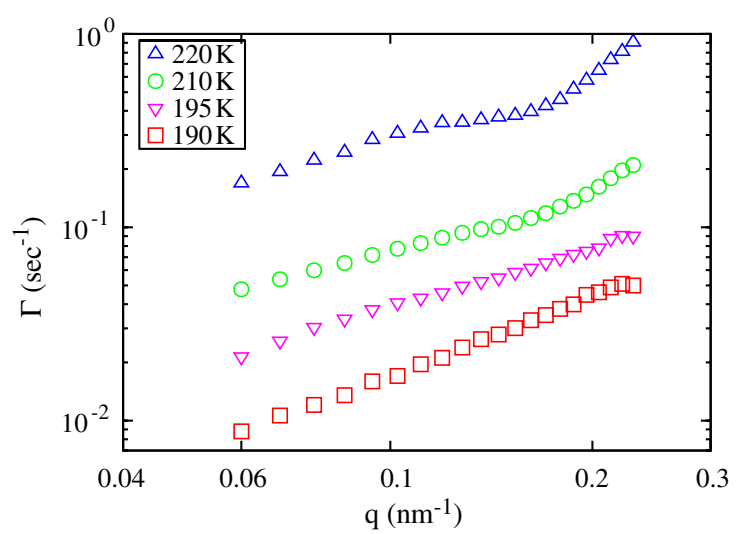

FIG. 5 (color online). $\quad \Gamma(q)$ for the concentrated $(\phi \sim 20 \%)$ suspension of $16 \mathrm{~nm}$ silica particles determined by multispeckle XPCS. The figure illustrates the disappearance of the dip in $\Gamma$ at $q=0.17 \mathrm{~nm}^{-1}$ ("de Gennes narrowing") at low temperatures.

istic frequency $\Gamma_{0}$ and the particles develop a strong tendency to move ballistically $(\alpha=1)$, at least for the shorttime decay of $f(t)$ which is discussed here. The average step size $\delta$ is rather unaffected by the transition and hence no indication of a diverging length scale [26] is present in the data. In addition, we observe cooperative behavior of probe particles in a concentrated suspension. We relate the observations to changed solvent properties and possibly elasticlike stress relaxations of the supercooled liquid. However, the dynamic behavior reported here is fundamentally different from earlier reported studies on jammed systems where $\gamma \rightarrow 3 / 2$ for $q \rightarrow 0$ was found. In the near vitreous state it is well known that the translational diffusion is decoupled from the viscosity. Computer simulations have evidenced that jumping between local minima of the potential energy landscape gets important at temperatures around $1.2 T_{g}$ [1] involving the cooperative motion [27] of larger regions and dynamical heterogeneity. Our experiments show that a dilute colloidal suspension of nanoparticles in a supercooled solvent develops hyperdiffuse behavior at $\sim 215 \mathrm{~K}\left(1.26 T_{g}\right)$ but the detailed connection with the glass transition remains an open question. Further studies are planned, e.g., to elucidate possible influences of the particle size and morphology [28].

R. Steinmann and the ESRF sample environment group are acknowledged for the SAXS cryostat, and we thank C. Ponchut, J. Clement, J.-M. Rigal, and E. Papillon for help with the Medipix-2 detector. A.C. gratefully acknowledges a grant from the Italian MIUR (PRIN 2005, No. 2005027011).

*amadsen@esrf.fr

[1] P. G. Debenedetti and F. H. Stillinger, Nature (London) 410, 259 (2001).
[2] W. Götze and L. Sjögren, Transp. Theory Stat. Phys. 24, 801 (1995).

[3] F. Sciortino, J. Stat. Mech. (2005) P05015.

[4] C. A. Angell, J. Phys. Chem. Solids 49, 863 (1988).

[5] S. Sastry, P. G. Debenedetti, and F. H. Stillinger, Nature (London) 393, 554 (1998).

[6] M. T. Cicerone and M.D. Ediger, J. Chem. Phys. 104, 7210 (1996).

[7] W. Huang and R. Richert, Philos. Mag. 87, 371 (2007).

[8] G. Grübel, A. Robert, and D. L. Abernathy, AIP Conf. Proc. 469, 158 (1999).

[9] A. Madsen, O. Konovalov, A. Robert, and G. Grübel, Phys. Rev. E 64, 061406 (2001).

[10] $T_{g}$ values were obtained with differential scanning calorimetry using a Perkin-Elmer Pyris Diamond calorimeter; calorimetric upscans were measured with a rate of $10 \mathrm{~K} / \mathrm{min}$, after rapid cooling $(500 \mathrm{~K} / \mathrm{min})$ of the sample to liquid nitrogen temperature. As usual, $T_{g}$ was identified from the stepwise increase of the specific heat.

[11] X. Llopart et al., IEEE Trans. Nucl. Sci. 49, 2279 (2002).

[12] D. Lumma, L. Lurio, S. G. M. Mochrie, and M. Sutton, Rev. Sci. Instrum. 71, 3274 (2000).

[13] G. Williams and D. C. Watts, Trans. Faraday Soc. 66, 80 (1970).

[14] C. Z.-W. Liu and I. Oppenheim, Phys. Rev. E 53, 799 (1996).

[15] L. Cipelletti, S. Manley, R. C. Ball, and D. A. Weitz, Phys. Rev. Lett. 84, 2275 (2000).

[16] M. Bellour, A. Knaebel, J. L. Harden, F. Lequeux, and J.-P. Munch, Phys. Rev. E 67, 031405 (2003).

[17] P. Falus, M. A. Borthwick, S. Narayanan, A. R. Sandy, and S.G. J. Mochrie, Phys. Rev. Lett. 97, 066102 (2006).

[18] A. Robert, E. Wandersman, E. Dubois, V. Dupuis, and R. Perzynski, Europhys. Lett. 75, 764 (2006).

[19] A. Fluerasu, A. Moussaïd, A. Madsen, and A. Schofield, Phys. Rev. E 76, 010401(R) (2007).

[20] J.-P. Bouchaud and E. Pitard, Eur. Phys. J. E 6, 231 (2001).

[21] L. Cipelletti, L. Ramos, S. Manley, E. Pitard, D. A. Weitz, E. E. Pashkovski, and M. Johansson, Faraday Discuss. 123, 237 (2003).

[22] J.-P. Bouchaud and A. Georges, Phys. Rep. 195, 127 (1990); E. W. Montroll and G. H. Weiss, J. Math. Phys. (N.Y.) 6, 167 (1965).

[23] R. Metzler and J. Klafter, Phys. Rep. 339, 1 (2000).

[24] B. J. Berne and R. Pecora, Dynamic Light Scattering (Wiley, New York, 1976).

[25] A. Duri and L. Cipelletti, Europhys. Lett. 76, 972 (2006).

[26] L. Berthier, G. Biroli, J.-P. Bouchaud, L. Cipelletti, D. El Masri, D. L'Hote, F. Ladieu, and M. Pierno, Science 310, 1797 (2005).

[27] E. Vidal Russel and N. E. Israeloff, Nature (London) 408, 695 (2000).

[28] R. Zangi, S. A. Mackowiak, and L. J. Kaufman, J. Chem. Phys. 126, 104501 (2007). 